\title{
Movement Observation Improves Early Consolidation of Motor Memory
}

\author{
Xue Zhang, ${ }^{\star}$ Toon T. de Beukelaar, ${ }^{\star}$ Jessy Possel, Marie Olaerts, Stephan P. Swinnen, Daniel G. Woolley, \\ and Nicole Wenderoth \\ Motor Control Laboratory, Research Centre for Movement Control and Neuroplasticity, Department of Biomedical Kinesiology, K. U. Leuven, 3001 \\ Heverlee, Belgium
}

Practicing a motor task can induce neuroplastic changes in the human primary motor cortex (M1) that are subsequently consolidated, leading to a stable memory trace. Currently, little is known whether early consolidation, tested several minutes after skill acquisition, can be improved by behavioral interventions. Here we test whether movement observation, known to evoke similar neural responses in M1 as movement execution, can benefit the early consolidation of new motor memories. We show that observing the same type of movement as that previously practiced (congruent movement stimuli) substantially improves performance on a retention test 30 min after training compared with observing either an incongruent movement type or control stimuli not showing biological motion. Differences in retention following observation of congruent, incongruent, and control stimuli were not found when observed $24 \mathrm{~h}$ after initial training and neural evidence further confirmed that, unlike motor practice, movement observation alone did not induce plastic changes in the motor cortex. This time-specific effect is critical to conclude that movement observation of congruent stimuli interacts with training-induced neuroplasticity and enhances early consolidation of motor memories. Our findings are not only of theoretical relevance for memory research, but also have great potential for application in clinical settings when neuroplasticity needs to be maximized.

\section{Introduction}

Performing repetitive movements for several minutes induces neural reorganization within the human motor cortex, a phenomenon known as use-dependent plasticity (Classen et al., 1998). Similar neuroplastic changes can be induced through movement observation. For example, observing repetitive rapid thumb movements for $30 \mathrm{~min}$ alters the cortical representation of muscles in favor of the agonist of the observed movement direction (Stefan et al., 2005). This effect is further enhanced when movement observation is combined with physical practice (Celnik et al., 2006, 2008; Stefan et al., 2008). Neural changes due to use-dependent plasticity involve the primary motor cortex (M1) and can be quantified by transcranial magnetic stimulation (TMS) (Classen et al., 1998; Perez et al., 2007; Rosenkranz et al., 2007a,b). Physical motor training leads to larger motor-evoked potentials (MEPs) for a given stimulation intensity, indicating an increase in corticomotor excitability. This effect is most likely the result of increased synaptic efficiency brought about by a mechanism similar to long-term potentiation (Stefan et al., 2006; Rosenkranz et al.,

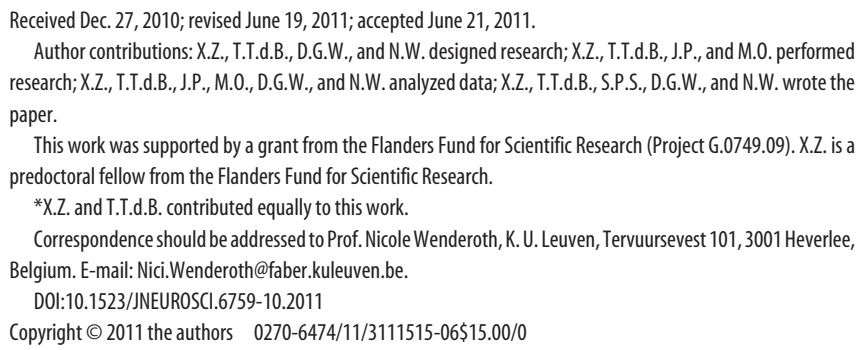

2007a). Muellbacher et al. (2002) showed that disrupting M1 function by low-frequency, repetitive TMS immediately after learning reduces behavioral retention, but has no effect when applied $6 \mathrm{~h}$ after training or over other cortical areas. These findings indicate that the human M1 plays a crucial role in the consolidation of new motor memories and that its involvement is most important during an early time window lasting only hours after training.

Here we examine the behavioral and neural effects of movement observation when performed during the early consolidation period immediately following motor training. Subjects first practiced rapid thumb flexion movements for $3 \mathrm{~min}$; followed immediately by $7 \mathrm{~min}$ of observing either congruent flexion movements, incongruent extension movements, or control stimuli not showing biological motion; followed by a retention test. We hypothesized an improvement in retention after observation of congruent movements compared with incongruent movements or control stimuli. To test whether the influence of movement observation on motor consolidation is specific to a short time window after training, control groups followed an identical protocol except that the observation task and retention test were performed the next day. We predicted that the three movement observation conditions would not differentially affect retention when performed after a $24 \mathrm{~h}$ break. Neural changes to the thumb flexor representation in M1 were recorded by TMS before motor training and immediately after movement observation. Based on previous research applying a similar approach (Rosenkranz et al., 2007a), we expected an increase in corticomotor excitability as a consequence of training, which might also depend on whether congruent or incongruent movements were observed. 
A Congruent video

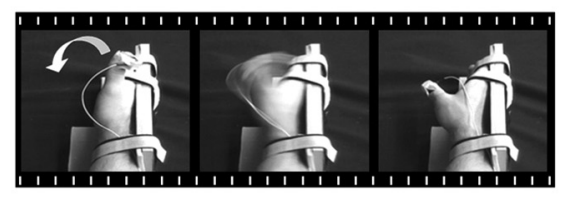

B Incongruent video

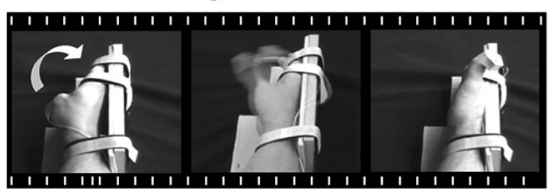

C Control video

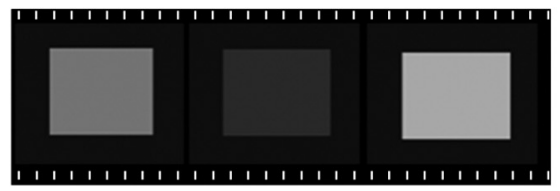

D

immed-Groups

\begin{tabular}{|c|c|c|c|c|c|}
\hline & & Congruent video (A) & & Retention- & Overtraining \\
10 curve & Training & OR & IO curve & Test & $(4 \times 20$ flexion $)$ \\
$21 \mathrm{~min}$ & $\begin{array}{c}(3 \times 20 \text { flexion }) \\
\text { T1 T2 T3 }\end{array}$ & $\begin{array}{c}\text { Incongruent video (B) } \\
\text { OR }\end{array}$ & 21 min & 20 flexion & T4 T5 T6 T7 \\
& & Control video (C) & & RT & \\
\hline
\end{tabular}

\section{E $\quad 24 \mathrm{hr}-G r o u p s$}

\begin{tabular}{|c|c|c|c|c|c|c|c|c|}
\hline $\begin{array}{l}10 \text { curve } \\
21 \mathrm{~min}\end{array}$ & $\begin{array}{c}\text { Training } \\
(3 \times 20 \text { flexion })\end{array}$ & 10 curve & & $\begin{array}{l}10 \text { curve } \\
21 \mathrm{~min}\end{array}$ & $\begin{array}{l}\text { Congruent video }(\mathrm{A}) \\
\text { OR } \\
\text { Incongruent video (B) }\end{array}$ & 10 curve & \begin{tabular}{|} 
Retention- \\
Test \\
20 flexion
\end{tabular} & $\begin{array}{l}\text { Overtraining } \\
\text { (4×20 flexion) }\end{array}$ \\
\hline & $\begin{array}{lll}\text { T1 } & \text { T2 } & \text { T3 }\end{array}$ & & $\stackrel{\text { a }}{\text { ᄃ }}$ & & $\begin{array}{c}\text { OR } \\
\text { Control video (C) }\end{array}$ & & $\begin{array}{c}20 \text { flexion } \\
\text { RT }\end{array}$ & $\begin{array}{llll}\text { T4 } & \text { T5 } & \text { T6 } & \text { T7 }\end{array}$ \\
\hline
\end{tabular}

Figure 1. $\boldsymbol{A}-\boldsymbol{C}$, Experimental protocol. Subjects observed three different types of videos showing thumb flexions congruent to the practiced task $(\boldsymbol{A})$, thumb extensions incongruent to the practiced task $(\boldsymbol{B})$, or a control not showing biological movements $(\boldsymbol{C}) \cdot \boldsymbol{D}, \boldsymbol{E}$, Three groups observed the different videos immediately after motor practice (immed-Groups, $\boldsymbol{D})$ and three more the next day (24 h-Groups, $\boldsymbol{E})$.

\section{Materials and Methods}

Subjects. Forty-eight ( 24 females; mean age, $22.88 \pm 3.36$ years) healthy, naive, right-handed subjects participated in this study. The experiments were approved by the local Ethics Committee for Biomedical Research at the K. U. Leuven and conform to the Declaration of Helsinki (1964).

General setup. Subjects sat in a comfortable chair with their right forearm resting in a neutral position with the thumb fully relaxed. MEPs were recorded from right musculus opponens pollicis $(\mathrm{OP})$ with surface electromyography (EMG; Mespec 8000; Mega Electronic). Two disposable $\mathrm{Ag}-\mathrm{AgCl}$ surface electrodes (Blue Sensor P-00-S; Ambu) were placed in a belly-tendon montage on the right OP. EMG data were sampled at 5000 $\mathrm{Hz}$ (CED Power 1401; Cambridge Electronic Design), amplified, bandpass filtered $(5-1000 \mathrm{~Hz})$, and stored on a PC for off-line analysis.

TMS measurements. TMS was performed with a figure-of-eight coil (loop diameter, $70 \mathrm{~mm}$ ) connected to a Magstim 200 stimulator (Magstim). The coil was held over the hotspot of OP (i.e., the location with the largest and most consistent MEPs) and with the optimal orientation for evoking a descending volley in the corticospinal tract. Rest motor threshold (RMT), defined as the lowest stimulus intensity eliciting MEPs $>50$ $\mu \mathrm{V}$ in at least five of 10 consecutive trials, was determined to the nearest $1 \%$ of maximum stimulator output. Corticomotor excitability was quantified by input-output curves (IO-curve), applying 10 stimulation intensities ranging from $90 \%$ to $180 \%$ RMT in steps of $10 \%$. The interstimulus interval varied randomly between 5 and $9 \mathrm{~s}$ and a block of 50 MEPs was collected in $\sim 6 \mathrm{~min}$. For each IO-curve, we recorded $15 \mathrm{MEPs}$ per intensity in a pseudorandom order. Breaks of 2 min were included after each block and the whole procedure lasted $\sim 24 \mathrm{~min}$. Subjects closed their eyes and relaxed their hand muscles, which was closely monitored on the basis of the background EMG (bgEMG).

Motor training. Subjects were trained to flex their thumb as fast as possible while their right hand was firmly fixed. 3D kinematics of the thumb movements were measured using a Polhemus Fastrak system (sampling rate, $120 \mathrm{~Hz}$; spatial resolution, $0.0006 \mathrm{~cm}$ ) with the sensor taped onto the nail of the thumb. Subject performed one thumb movement every $3 \mathrm{~s}$ and on-line feedback was provided by displaying the peak velocity of the current trial as well as its evolution across training. To prevent fatigue, $60 \mathrm{~s}$ breaks were provided after each block of 20 movements. Subjects were continuously instructed to increase movement speed and were motivated by an experimenter who was blind to which group the subject belonged.

Movement observation. Subjects observed videos showing one of the following: (1) fast thumb flexion movements (FLEX), i.e., congruent with the motor task performed during training; (2) fast thumb extension movements (EXT), i.e., incongruent with the motor task; or (3) control stimuli consisting of a series of squares colored with different gray levels (CONT), i.e., not showing biological motion (Fig. $1 A-C)$. The thumb flexion and extension videos showed a highly trained actor from the first person perspective performing a fast thumb movement every $3 \mathrm{~s}$ in front of a black background. In total, 138 movements were shown. The control stimuli consisted of a series of 138 squares appearing for $2 \mathrm{~s}$ in front of a black background, with a new square presented once every 3 s. For all three tasks, we tested the alertness of subjects with an occasional oneback discrimination task. At random intervals, subjects had to compare the speed/color of the last observed movement/square to the one before and judge which of the two stimuli was faster/brighter. All groups performed the discrimination task significantly above chance level ( $85 \pm$ $7.9 \%)$. Subjects were constantly reminded to always keep their thumb relaxed during movement observation, which was monitored visually by the experimenter.

Study design. Subjects were randomly assigned to one of six groups (four males and four females per group) (Fig. 1). In three groups (Fig. $1 D$ ), the observation task was performed immediately following motor training. The first IO-curve was recorded at the beginning of the experiment. Then subjects performed three blocks of motor training (T1, T2, T3), with each block consisting of 20 thumb flexion movements. Immediately after training, subjects watched congruent movements (immedFLEX), incongruent movements (immed-EXT), or the control stimuli (immed-CONT). After the observation intervention, a second IO-curve was recorded. During the 2 min breaks of the IO-curve procedure and immediately after the IO-curve was completed, $1.5 \mathrm{~min}$ fragments of the intervention movies were shown. Immediately after the final movie fragment ( $\sim 30$ min after training), we tested retention (RT) of motor per- 
A

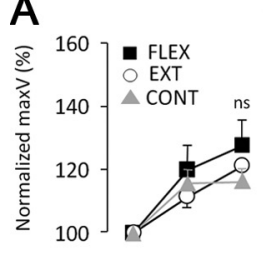

$\begin{array}{lll}\mathrm{T} 1 & \mathrm{~T} 2 & \mathrm{~T} 3\end{array}$
immed-Groups

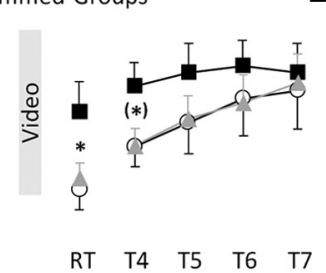

B

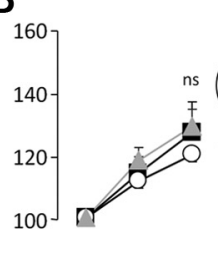

$24 \mathrm{hr}-$ Groups

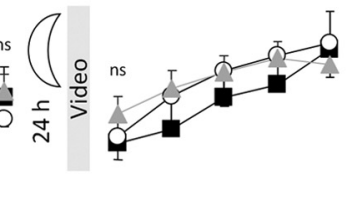

RT T4 T5 T6 T7
D $24 \mathrm{hr}$-Train

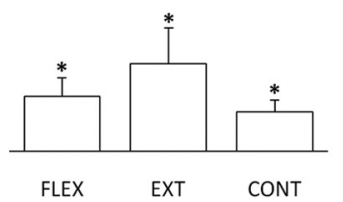

G

$\mathbf{F}$

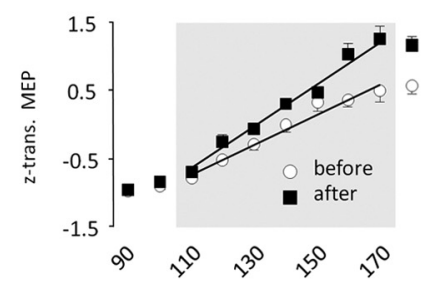

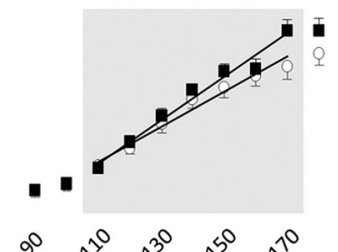

E $\quad 24 \mathrm{hr}$-Video

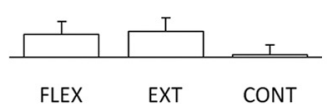

H immed-CONT

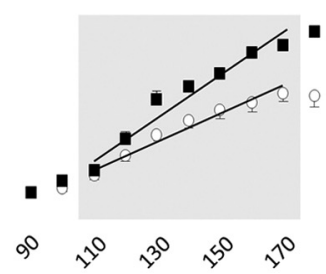

Figure 2. Behavioral and neural results. $\boldsymbol{A}, \boldsymbol{B}$, Maximal flexion velocity (maxV) normalized to $\mathrm{T} 1$ (corresponding to $100 \%$ ) is shown when flexion (FLEX), extension (EXT), or control (CONT) movies were observed immediately after training $(\boldsymbol{A})$ or the next day $(\boldsymbol{B})$. Significant differences between groups are indicated by ${ }^{*}(p<0.05)$ and a trend toward significance $(p=0.06)$ by $\left({ }^{*}\right)$. ns, Not significant. $\boldsymbol{C}-\boldsymbol{E}$, Median changes in the slopes of I0-curves ( $\Delta$ Slope) measured before and after physical training, immediately followed by observing different videos (immed) (C), physical training alone ( $24 \mathrm{hr}$-Train, first day) (D), and movement observation alone (24 hr-Video, second day) $(\boldsymbol{E})$. $\Delta$ Slope values differing significantly from 0 are indicated by $*(p<0.05)$ as revealed by single-sample $t$ tests. $\boldsymbol{F}-\boldsymbol{H}, 10$-curves of the immediate groups showing z-transformed MEP amplitudes before and after training plus movement observation. Trend lines indicate the linear fit applied to the range from $110 \%$ to $170 \%$ RMT as indicated by the shaded area. Vertical bars indicate SEs.

formance during one block of 20 thumb flexion movements, which was followed by four more training blocks (T4, T5, T6, T7).

Three more groups (Fig. $1 \mathrm{E}$ ) followed the same protocol but had a $24 \mathrm{~h}$ break (including sleep) between initial motor training and performing the movement observation task. On the first day, an IO-curve was recorded, followed by motor training (three blocks of 20 thumb flexion movements), followed by another IO-curve. An IO-curve was recorded at the start of the second day, then subjects watched either congruent movements (24 h-FLEX), incongruent movements (24 h-EXT), or the control stimuli (24 h-CONT), followed by another IO-curve. Again, short video fragments were shown during the breaks and upon completion of the TMS measurement. Finally, all subjects performed the RT and four more training blocks (T4-T7).

Data analysis. Motor performance was quantified by the peak velocity of the thumb movement. The absolute $3 \mathrm{D}$ velocity was calculated from kinematics by $V_{\mathrm{i}}=\left[\left(x_{\mathrm{i}}-x_{\mathrm{i}-1}\right)^{2}+\left(y_{\mathrm{i}}-y_{\mathrm{i}-1}\right)^{2}+\left(z_{\mathrm{i}}-z_{\mathrm{i}-1}\right)^{2}\right]^{1 / 2} /\left(t_{\mathrm{i}}-t_{\mathrm{i}-1}\right)$, whereby $x, y$, and $z$ denote the displacement in the three dimensions, $t$ the time, and $i$ the index of the actual data point. The velocity data were low-pass filtered (second-order Butterworth, $30 \mathrm{~Hz}$ cutoff frequency) and the maximum velocity $(\operatorname{maxV})$ was extracted for each trial and averaged within each block of 20 trials. Figures show normalized maxV values that are expressed as a percentage of the first block.

For each TMS pulse, MEP size was determined by its peak-to-peak amplitude and the bgEMG was quantified by the root mean square error of the EMG signal in an interval between 10 and 110 ms before TMS stimulation. For each subject, mean and standard deviation of the bgEMG scores were computed and trials with bgEMG larger than the mean plus 2.5 SDs were removed from the analysis. Trials with exceptionally large MEP amplitudes were also excluded, i.e., when the peak-to-peak amplitude exceeded Q3 + $1.5 \times$ (Q3 - Q1), with Q1 and Q3 being the first and third quartile computed for the MEPs within each intensity. According to these criteria, $92 \pm$ $0.31 \%$ of the trials were subjected to further analyses. For the premeasurement, all groups exhibited comparable MEP amplitudes for low intensities; however, in response to high TMS intensities ( $>150 \%$ RMT) the immedCONT group exhibited significantly larger MEPs than the immed-FLEX group ( $p<$ 0.035, between-group ANOVAs). Therefore, we analyzed relative changes of the IO-curve from pretraining to posttraining. Previous studies (Rosenkranz et al., 2007a,b) have demonstrated that motor training increases the steepness of the IO-curve slope. For each subject, the slope was estimated from the linear part of the IO-curve between $110 \%$ and $170 \%$ RMT (mean $r^{2}=0.87 \pm 0.09$ ). $\Delta$ Slope was calculated as the slope difference between the IO-curves measured before and after training and was expressed as a percentage $\left[\Delta\right.$ Slope $=\left(\right.$ Slope $_{\text {pre }}$ Slope $\left._{\text {post }}\right) /$ Slope $\left._{\text {pre }} \times 100\right)$. For completeness, we also report changes in MEP amplitudes after averaging across all stimulation intensities for the pre- and post-IO-curves, as well as the percentage increase of MEP amplitudes from pre- to post-IO-curves.

Statistics. Changes in behavioral performance, as quantified by the normalized maxV values, were analyzed by preplanned comparisons calculated in the context of an ANOVA model with the within-factor session (T1-T3, RT, T4-T7) and between-factor group (immedFLEX, immed-EXT, immed-CONT). Identical analyses were performed for the late intervention groups.

Simple $t$ tests were used to determine whether the $\Delta$ Slope of each group differed significantly from 0 . Between-group differences were tested by an ANOVA with the factor group. The same statistics were calculated separately for the first and the second day of the $24 \mathrm{~h}$ groups. Post hoc comparisons were Bonferroni corrected with $\alpha=0.05$. Group results are reported with means and standard errors.

\section{Results}

\section{Behavioral results}

Observing congruent flexion movements immediately after training (immed-FLEX) resulted in significant performance savings at RT. By contrast, performance returned to baseline levels after observing incongruent extension movements (immedEXT) or control stimuli (immed-CONT). Group differences were not found when the different observation conditions were performed $24 \mathrm{~h}$ after training.

All early intervention groups improved during the first three blocks of motor training (Fig. $2 A$ ), indicated by a significant increase in normalized $\operatorname{maxV}\left(F_{(1,21)} \geq 9.26, p<0.01\right)$ and similar performance levels at T3. After observing the videos, the immed-FLEX group slightly improved performance at RT compared with T3, whereas the immed-EXT and immed-CONT groups exhibited a significant drop in performance $\left(F_{(1,21)} \geq\right.$ $4.41, p<0.05)$. Furthermore, performance of the immed-EXT and immed-CONT groups at RT was reduced to near baseline 
Table 1. Mean and standard deviation of age, performance on the attention test, RMT, the group median of the absolute MEP amplitudes of the pre- and post-10-curves, and the percentage increase in MEP amplitudes from pretraining to posttraining

\begin{tabular}{|c|c|c|c|c|c|c|}
\hline & Age (years) & Attention (\% correct) & RMT (\%) & MEP $(\mathrm{mV})$ pretraining & MEP (mV) posttraining & Increase (\%) \\
\hline immed-FLEX & $23 \pm 2$ & $88.00 \pm 9$ & $39.75 \pm 5$ & $0.60 \pm 0.82$ & $0.76 \pm 0.39$ & $43.78 \pm 30.41^{*}$ \\
\hline immed-EXT & $23 \pm 4$ & $82.64 \pm 8$ & $39.25 \pm 5$ & $0.62 \pm 0.88$ & $0.76 \pm 1.51$ & $11.69 \pm 73.18$ \\
\hline immed-CONT & $24 \pm 4$ & $90.97 \pm 5$ & $44.25 \pm 8$ & $2.28 \pm 1.83$ & $2.99 \pm 1.37$ & $37.88 \pm 52.37^{*}$ \\
\hline 24 h-FLEX day 1 & $20 \pm 2$ & & $38.38 \pm 6$ & $1.68 \pm 1.42$ & $2.19 \pm 1.48$ & $11.97 \pm 19.09^{*}$ \\
\hline 24 h-EXT day 1 & $22 \pm 1$ & & $44.5 \pm 6$ & $1.26 \pm 0.70$ & $2.30 \pm 0.76$ & $74.92 \pm 71.58^{*}$ \\
\hline 24 h-CONT day 1 & $26 \pm 3^{\#}$ & & $44.38 \pm 5$ & $1.36 \pm 0.85$ & $1.60 \pm 1.62$ & $32.81 \pm 31.09^{*}$ \\
\hline 24 h-FLEX day 2 & & $80.56 \pm 7$ & $38.13 \pm 7$ & $1.62 \pm 1.43$ & $2.23 \pm 1.34$ & $11.47 \pm 20.81$ \\
\hline 24 h-EXT day 2 & & $81.94 \pm 10$ & $44.00 \pm 5$ & $1.75 \pm 0.71$ & $1.84 \pm 0.70$ & $13.34 \pm 29.47$ \\
\hline $24 \mathrm{~h}-\mathrm{CONT}$ day 2 & & $84.03 \pm 9$ & $44.13 \pm 6$ & $1.38 \pm 0.52$ & $1.41 \pm 0.37$ & $0.11 \pm 16.92$ \\
\hline
\end{tabular}

"The $24 \mathrm{~h}$-CONT was significantly older than the $24 \mathrm{~h}$-FLEX group $(p<0.01) .{ }^{*}$ Significant percentage increase in the mean MEP amplitude from pretraining to posttraining ( $p<0.05$, single-sample $t$ test).

levels with normalized maxV not differing significantly from $\mathrm{T} 1$ $\left(F_{(1,21)} \leq 1.45, p \geq 0.24\right)$. We also found a significant group (immed-Flex, immed-EXT) $\times$ time $(\mathrm{T} 3, \mathrm{RT})$ interaction $\left(F_{(1,21)}=\right.$ $10.6, p<0.003)$, confirming that retention was significantly better following observation of congruent movements compared with incongruent movements. Note that the performance advantage of the immed-FLEX group was still substantial at T4 and nearly reached significance when compared with the other groups $\left(F_{(1,21)} \geq 3.94, p \leq 0.06\right)$. During subsequent training blocks T4-T7, all groups demonstrated further significant improvement $\left(F_{(1,21)} \geq 4.34, p<0.05\right)$, reaching a similar level of performance at T7. However, the normalized $\operatorname{maxV}$ of the immed-FLEX group at T4 did not differ significantly from T7 $\left(F_{(1,21)}=0.58, p=0.455\right)$, indicating a rapid plateau in performance. By contrast, it took much longer for the immed-EXT and immed-CONT groups to reach the final performance level, highlighted by the fact that T5 was still significantly different from T7 for both groups $\left(F_{(1,21)} \geq 8.6, p<0.01\right)$.

The 24 h groups (Fig. $2 B$ ) improved significantly during initial training $\left(F_{(1,21)} \geq 24.64, p<0.0001\right)$, reaching comparable performance levels at T3. Subjects performed worse at RT compared with T3, but still better than at T1 $\left(F_{(1,21)} \geq 4.97, p<0.05\right)$. This suggests that at least some of the performance improvement resulting from training on day 1 was retained $1 \mathrm{~d}$ later. Most importantly, there were no significant differences between groups at RT $\left(F_{(1,21)} \leq 0.63, p \geq 0.54\right)$ or during the training blocks T1-T7 $\left(F_{(1,21)} \leq 1.7, p \geq 0.2\right)$. Thus, movement observation performed $24 \mathrm{~h}$ after training did not influence behavior at RT or on subsequent training trials.

\section{Corticomotor excitability}

In the immediate observation groups, IO-curves were measured before motor training and after movement observation was completed; thus, changes reflect the combined effect of both tasks. While IO-curves were steeper (Fig. 2C,F-H) and mean MEP amplitudes were higher (Table 1 ) in general after motor training plus movement observation, a significant increase in slope was only found in the immed-FLEX $(t=3.41, p<0.05)$ and immedCONT $(t=2.75, p<0.05)$ groups, and not in the immed-EXT group $(t=1.66, p=0.14)$. A comparison of the change in slope of IO-curves across groups did not reveal any significant differences.

IO-curves for the $24 \mathrm{~h}$ groups were measured immediately before and after training on day 1 . We found a significant increase in slope $(p<0.05)$ and MEP amplitudes (Table 1$)$ for all groups (Fig. 2D). IO-curves on day 2 were measured before and after observation only, which had only a small and nonsignificant influence on the slope of the IO-curves (Fig. 2 E). For both days, there were no significant differences in $\Delta$ Slope between groups.

\section{Discussion}

Movement observation performed immediately after practicing fast thumb flexion movements significantly improved retention 30 min after training when subjects observed congruent flexion movements, but not when they observed incongruent extension movements or control stimuli. No such differences in retention performance were observed when movement observation was performed $24 \mathrm{~h}$ after training. These results indicate that movement observation did not influence recall or motor performance per se, but specifically the consolidation of early motor memories. Furthermore, we found a significant increase in the slope of IO-curves following both motor training performed alone and in combination with the observation tasks, the notable exception being the incongruent movement observation group. Importantly, movement observation $24 \mathrm{~h}$ after motor training induced only small, insignificant changes in the IO-curves, indicating that 7 min of movement observation alone was insufficient to induce a measurable degree of cortical reorganization.

Performing rapid thumb movements repeatedly for several minutes is a well established model to study use-dependent plasticity within the human motor cortex. Using this paradigm, it has previously been shown that movement observation can induce neuroplastic changes in the M1 similar to physical practice (Stefan et al., 2005, 2008; Celnik et al., 2006, 2008). Here we demonstrate for the first time that movement observation can have a beneficial effect on the early consolidation of a motor memory. This finding extends previous research by showing that observation training not only induces neural reorganization, but that it can influence motor performance to an extent that is functionally relevant.

Retention can be quantified either in relation to baseline performance at the beginning of training (T1) or in relation to the final performance at the end of the initial training session (T3). Observing congruent movements led to significantly better retention, which resulted in a small performance increase of $2.73 \%$ compared with T3 and a significant increase of $31.02 \%$ compared with T1. In contrast, observing extension movements resulted in a significant performance decrement of $-12.24 \%$ from T3 to RT, and only a small $6.11 \%$ performance saving compared with T1. This finding was further confirmed by a significant group $\times$ time interaction effect. We also tested a control group observing stimuli not showing biological motion to further understand whether the effect of observing congruent movements was really beneficial, or whether observing incongruent movements was detrimental. A small performance drop of $-5.14 \%$ from T3 to RT was found in the control group; however, the $(\mathrm{T} 3, \mathrm{RT}) \times$ group interaction did not reach significance when compared with either 
observation group. When comparing RT performance to T1, the control group exhibited only insignificant behavioral savings. Together, our data support the conclusion that observing the same movements as those just practiced is beneficial for subsequent motor performance. This effect was relatively long lasting, because it was not only found across the 20 trials of the retention test, but also during the subsequent block (for which group differences approached statistical significance). Consequently, performance of the immed-FLEX group reached plateau much earlier.

Importantly, when subjects performed the observation tasks $24 \mathrm{~h}$ after practice, all groups performed worse than at T3 but exhibited a significant improvement when compared with T1. This indicates that after a $24 \mathrm{~h}$ break including sleep, they maintained some but not all of the improvement gained during motor training on the first day (Walker and Stickgold, 2004). While larger performance savings between days were reported by Rosenkranz et al. (2007a), subjects in that study were provided with substantially more practice at day 1 ( 240 vs 60 movements). Our rational for providing limited practice was that we wanted subjects to reach a performance level corresponding to $50 \%$ of the gain achievable within one session, which would allow for both performance improvements and decrements following movement observation. Indeed, this was the case as performance at T3 was approximately midway between T1 and the plateau at T7. Note also that the general learning capacity was similar across groups. The most important finding of the $24 \mathrm{~h}$ groups was that when the observation task was performed the day after physical practice, it did not result in behavioral differences across groups. Thus, our movement observation training was ineffective in influencing performance when not immediately preceded by physical practice. This also differs from previous studies reporting a significant effect of movement observation when it was not preceded by training (Stefan et al., 2005, 2008). The differential results might have been caused by the longer observation period used in previous work (1800 stimuli across a period of $30 \mathrm{~min}$ vs 7 min showing 138 movements in this study) and because Stefan et al. $(2005,2008)$ measured neural outcome parameters rather than motor performance.

It is well known that increasing the speed of ballistic thumb movements due to repetitive training is accompanied by increased corticomotor excitability of the involved muscle (Stefan et al., 2005; Rosenkranz et al., 2007). Additional experiments further suggested that corticomotor facilitation most likely resulted from increased synaptic efficiency within M1 driven by a mechanism similar to long-term potentiation (Bütefisch et al., 2000; Rosenkranz et al., 2007a,b). Our data are in agreement with the finding of increased excitability after learning as IO-curves became significantly steeper when training was either performed alone ( 24 h groups, day 1 ) or immediately followed by observing the congruent flexion movements or the control movie (immediate groups). Interestingly, the changes in IO-curve slope were larger for the immed-FLEX group than for the immed-EXT group, with the latter not changing significantly from pretraining to posttraining. However, we found no significant effects when comparing the groups directly. The lack of significant betweengroup effects might result, at least partly, from the high variability of the IO-curve measurements that was not only present for the immediate, but also for the $24 \mathrm{~h}$ groups. The $24 \mathrm{~h}$-EXT group exhibited a significantly larger increase in slope compared with the $24 \mathrm{~h}$-CONT group. Factors contributing to this high interindividual variability concerning learning might be differential sensitivity to TMS (Wassermann, 2002), variable cortisol levels (Sale et al., 2008), or genetic factors (Missitzi et al., 2011) beyond our control, but that might have biased the IO-curve results given the relatively small sample size.

Moreover, one has to note that the changes in IO-curves reflected not only the influence of movement observation, but also the influence of the preceding training, which is known to facilitate corticomotor excitability in M1. We refrained from measuring another IO-curve between physical practice and movement observation, since pilot work indicated that movement observation induced more stable effects when provided shortly after training but not after a 20 min delay (our unpublished pilot data). In a previous study, repetitive TMS was applied to M1 after use-dependent plasticity had been induced (Muellbacher et al., 2002). Similar to our results, IO-curves were reported to be steeper after motor training even though behavioral retention was significantly perturbed. This indicates that, even though corticomotor facilitation of M1 reflects learning at the neural level, it might not be ideal to characterize processes related to memory consolidation. Additional correlation analyses indicated that none of the corticomotor excitability measurements in M1 predicted any of the behavioral learning effects, consistent with the notion that other brain areas or spinal mechanisms might contribute to the behavioral expression of learning as well.

Currently, it is not clear which neural mechanisms might have mediated the difference between observing congruent versus incongruent movements during early consolidation. In humans, movement observation activates premotor areas including ventral premotor cortex which, in turn, facilitates M1. Interestingly, for natural movements, M1 facilitation due to observation is muscle-specific, time-locked to the visual stimuli, and mimics the force requirements of the actual movement, although behavioral effects are typically weaker and less generalized following movement observation compared with movement execution (Porro et al., 2007). Thus, observing the congruent flexion movements most likely activated M1 in a similar, albeit weaker, manner as physical practice, which was beneficial for motor consolidation. This is a new finding and potentially relevant in the context of neurorehabilitation, for example after stroke, as movement observation is an easy-toapply and cost-efficient intervention that might increase the efficiency of rehabilitation training.

\section{References}

Bütefisch CM, Davis BC, Wise SP, Sawaki L, Kopylev L, Classen J, Cohen LG (2000) Mechanisms of use-dependent plasticity in the human motor cortex. Proc Natl Acad Sci U S A 97:3661-3665.

Celnik P, Stefan K, Hummel F, Duque J, Classen J, Cohen LG (2006) Encoding a motor memory in the older adult by action observation. Neuroimage 29:677-684.

Celnik P, Webster B, Glasser DM, Cohen LG (2008) Effects of action observation on physical training after stroke. Stroke 39:1814-1820.

Classen J, Liepert J, Wise SP, Hallett M, Cohen LG (1998) Rapid plasticity of human cortical movement representation induced by practice. J Neurophysiol 79:1117-1123.

Missitzi J, Gentner R, Geladas N, Politis P, Karandreas N, Classen J, Klissouras $\mathrm{V}$ (2011) Plasticity in human motor cortex is in part genetically determined. J Physiol 589:297-306.

Muellbacher W, Ziemann U, Wissel J, Dang N, Kofler M, Facchini S, Boroojerdi B, Poewe W, Hallett M (2002) Early consolidation in human primary motor cortex. Nature 415:640-644.

Perez MA, Wise SP, Willingham DT, Cohen LG (2007) Neurophysiological mechanisms involved in transfer of procedural knowledge. J Neurosci 27:1045-1053. 
Porro CA, Facchin P, Fusi S, Dri G, Fadiga L (2007) Enhancement of force after action observation: behavioural and neurophysiological studies. Neuropsychologia 45:3114-3121.

Rosenkranz K, Kacar A, Rothwell JC (2007a) Differential modulation of motor cortical plasticity and excitability in early and late phases of human motor learning. J Neurosci 27:12058-12066.

Rosenkranz K, Williamon A, Rothwell JC (2007b) Motocortical excitability and synaptic plasticity is enhanced in professional musicians. J Neurosci 27:5200-5206.

Sale MV, Ridding MC, Nordstrom MA (2008) Cortisol inhibits neuroplasticity induction in human motor cortex. J Neurosci 28:8285-8293.

Stefan K, Cohen LG, Duque J, Mazzocchio R, Celnik P, Sawaki L, Ungerleider
L, Classen J (2005) Formation of a motor memory by action observation. J Neurosci 25:9339-9346.

Stefan K, Wycislo M, Gentner R, Schramm A, Naumann M, Reiners K, Classen J (2006) Temporary occlusion of associative motor cortical plasticity by prior dynamic motor training. Cereb Cortex 16:376-385.

Stefan K, Classen J, Celnik P, Cohen LG (2008) Concurrent action observation modulates practice-induced motor memory formation. Eur J Neurosci $27: 730-738$

Walker MP, Stickgold R (2004) Sleep-dependent learning and memory consolidation. Neuron 44:121-133.

Wassermann EM (2002) Variation in the response to transcranial magnetic brain stimulation in the general population. Clin Neurophysiol 113:1165-1171. 